Pacific Journal of Mathematics

A CHARACTERIZATION OF THE LINEAR SETS SATISFYING 


\title{
A CHARACTERIZATION OF THE LINEAR SETS SATISFYING HERZ'S CRITERION
}

\author{
Haskell P. Rosenthal
}

Let $E$ be a closed subset of $\mathrm{T}$, the circle group, which we identify with the real numbers modulo $1 . E$ is said to satisfy Herz's criterion (briefly, $E$ satisfies $(H)$ ), if there exists an infinite set of positive integers $N$, such that

(*) for all integers $j$ with $0 \leqq j<N$, each of the numbers $j / N$ either belongs to $E$ or is distant by at least $1 / N$ from $E$.

The main theorem proved here, is that $E$ satisfies $(H)$ if and only if there exists a sequence of sets $F_{1}, F_{2}, \cdots$ with $E=\bigcap_{i=1}^{\infty} F_{i}$ and positive integers $N_{1}<N_{2}<\cdots$ satisfying the following properties for all $i$ :

(1) $N_{i}$ divides $N_{i+1}$ and $F_{i} \supset F_{i+1}$.

(2) $F_{i}$ is a finite union of disjoint closed intervals each of whose end points is of the form $j / N_{i}$ for some integer $j$.

(3) If for some integer $j, j / N_{i} \in F_{i}$, then $j / N_{i} \in F_{i+1}$.

The motivation for studying sets $E$ satisfying $(H)$ is the result of Herz (c.f. [1]) that all such sets satisfy spectral synthesis, and of course that the Cantor set is an example. (See also [2], Chapter IX).

Now suppose that $E=\bigcap_{i=1}^{\infty} F_{i}$, with $F_{i}$ and $N_{i}$ satisfying (1)-(3) for all $i$. It is then evident that $E$ satisfies $(H)$, since the numbers $N_{i}$ will satisfy $(*)$ for all $i$. Moreover, $E$ is obtained by a sort of disection procedure. Indeed, $F_{i+1}$ may be obtained from $F_{i}$ by removing from certain of the closed intervals $\left[j / N_{i},(j+1) / N_{i}\right]$ included in $F_{i}$, one or more open intervals of the form

$$
\left(\frac{l}{N_{i+1}}, \frac{q}{N_{i+1}}\right)
$$

where $j / N_{i} \leqq l / N_{i+1}<q / N_{i+1} \leqq(j+1) / N_{i}$.

The "only if" part of our main result is demonstrated following the proof of Theorem 4 below. The latter result is somewhat stronger than our main theorem, and enables us to show that certain sets fail to satisfy $(H)$ (in particular, the symmetric sets of ratio $\xi$, where $\xi$ is a rational number with $1 / \xi$ unequal to an integer. (C.f. [2], pp. 13-15 for the definition of these sets).

§1. Preliminaries. We identify the points of $\boldsymbol{T}$ with $[0,1)$, where addition and subtraction are taken modulo 1 . If $x$ and $y$ belong to $\boldsymbol{T}$, then the distance between them, $\rho(x, y)$, is defined to be the distance from $x-y$ to the closest integer on the real line. If $E$ 
is a subset of $\boldsymbol{T}$, then $\rho(x, E)$ is defined as $\inf _{f \in E} \rho(x, f)$.

Throughout this paper, $E$ shall refer to a closed proper nonempty subset of $\boldsymbol{T}$ and $\mathscr{N}$ shall denote the set of all positive integers $N$ satisfying $(*)$. (Thus if $E$ satisfies $(H), \mathscr{N}$ is an infinite set (and conversly)). Every variable " $N$ ", with or without sub or superscripts, refers to a member of $\mathscr{N}$, and every variable " $j$ " refers to an integer.

If $L$ and $M$ are positive integers, we write $L \mid M$ if there is an integer $q$ with $L q=M$.

Given a set $S$, “ $\sim S$ " denotes its complement.

Let $[x]$ be the greatest integer less than or equal to $x$. We remind the reader that if $U$ is a proper connected open subset of $\boldsymbol{T}$, there will exist unique real numbers $a<b \leqq a+1$, such that $0 \leqq b<1$, and such that $U=\{x-[x]: a<x<b\}$. We then define the length of $U$ to be $b-a$, with the left and right end points of $U$ being $a-[a]$ and $b$ respectively.

Definition. Let $x$ be a member of $E$ for which there exists a $j$ with $0 \leqq j<N$, such that $x=j / N$.

$x$ is called $N$-initial if $(j-1) / N \notin E$.

$x$ is called $N$-terminal if $(j+1) N \notin E$.

$x$ is called an $N$-end if $x$ is $N$-initial or $N$-terminal.

We note that if $x$ is $N$-initial ( $N$-terminal) then $x$ is a right (left) end point of a component of $\sim E$ of length at least $2 / N$. Indeed, if $x$ is $N$-initial, we may close a $j$ so that $x-(1 / N)=j / N$, and $j / N \notin E$. Hence the open interval $((j / N)-(1 / N),(j / N)+(1 / N))$ cannot contain any points of $E$, and of course $x=(j+1) / N$ belongs to $E$.

2. Our first result shows that if $E$ satisfies $(H)$, then the boundary points of components of $\sim E$ must be rational numbers.

Lemma 1. Let $U$ be a component of $\sim E$, of length $l$. Then if $N>1 / l$, the end points of $U$ are $N$-ends.

Proof. Let $x$ be the left end point of $U$. Then $x \in E$. Suppose it were false that $x=j / N$ for some $j$. There would then exist a $0 \leqq j<N$ such that $x \in(j / N,(j+1) / N)$. Since $(1 / N)<l$, we would have that $((i+1) / N \in U)$, so $(j+1) / N \notin E$. But

$$
\rho\left(\frac{j+1}{N}, E\right) \leqq \rho\left(\frac{j+1}{N}, x\right)<\frac{1}{N},
$$

a contradiction. Thus, there exists a $j, 0 \leqq j<N$, with $x=j / N$. But then $(j+1) / N \notin E$, since the length of $(j / N,(j+1) / N)$ is $1 / N<l$, hence $(j+1) / N \in U$. Thus, $x$ is $N$-terminal. The proof that the 
right end point of $U$ is $N$-initial is similar.

Our next task is to define certain sets that are finite unions of disjoint closed intervals, that approximate $E$. First, we note that if $x$ is $N$-initial, then $x$ is associated with a unique $N$-terminal number (possibly equal to $x$ ), as follows: let $k$ be the smallest integer $l$, with $0 \leqq l<N$, such that $x+(l+1) / N \notin E$. (Note that $l=N-2$ is such an integer.) Then $x+k / N$ is the uniquely determined $N$-terminal number.

Wed efine $I_{x}=[x, x+(k / N)]$ and $E_{N}=\bigcup\left\{I_{x}: x\right.$ is $N$-initial $\}$. If there do not exist any $N$-ends, set $E_{N}=\mathrm{T}$. Let $l_{1}$ be the maximum of the lengths of components of $\sim E$.

Then if $N>1 / l_{1}$, there will exist $N$-ends by Lemma 1 and hence $E_{N}$ will be a proper subset of $\boldsymbol{T}$. Of course, $I_{x} \cap I_{x \prime}=\phi$ for $x$ and $x^{\prime}$ different $N$-ends; so $E_{N}$ is a disjoint union of intervals with end points all of the form $j / N$.

Lemma 2. For all $N$ and $N^{\prime}, N^{\prime}<N$ implies $E_{N} \subset E_{N^{\prime}}$.

Proof. Let $N^{\prime}<N$ be fixed, and let $x$ be a fixed $N$-initial number. It follows directly from the definitions that $E \subset E_{N^{\prime}}$; thus since $x \in E$, there is a (unique) $N^{\prime}$-end $y$, such that $x \in I_{y}^{\prime}$, where $I_{y}^{\prime}=$ $[y, z]$, with $z$ the unique $N^{\prime}$-terminal number associated with $y$.

Now choose an integer $l$ with $0 \leqq l<N$ such that

$$
z \in\left[\frac{l}{N}, \frac{l+1}{N}\right) \text {. }
$$

Then $(l+1) / N \notin E$, since $(l+1) / N \in(z, z+1 / N)$. Thus we must have that $z=l / N$, or else $\rho(l / N, E) \leqq \rho(l / N, z)<1 / N$. Hence $z$ is $N$-terminal, and so it follows from the definition of $I_{x}$ that $I_{x} \subset I_{y}^{\prime}$.

Thus $E_{N} \subset \bigcup\left\{I_{y}^{\prime}: y\right.$ is $N^{\prime}$-initial $\}=E_{N}$.

Our last lemma enables us to obtain certain canonical members of $N$ crucial for the proof of Theorem 4 (whose proof also shows that the number $N / d$ below equals $q_{i}$, where $l_{i+1} \leqq \frac{1}{N}<l_{i}$ and $q_{i}, l_{i}$ are defined directly preceeding the statement of Theorem 4 ).

Lemma 3. Let $S_{N}=\{0 \leqq j<N: j / N$ is an $N$-end $\}$.

Let $d$ be a positive integer such that $d \mid N$ and $d \mid j$ for all $j \in S_{N}$. Then $(N / d) \in \mathscr{N}$.

Proof. We may and shall assume that $d>1$. Put $M=N / d$, and let $l$ be an integer with $0 \leqq l<M$, such that $l / M \notin E$. It remains 
for us to show that $\rho(l / M, E) \geqq 1 / M$. If this is not the case, then either $\{(l-1) / M, l / M)$ or $\{l / M,(l+1) / M)$ contains a point of $E$. Suppose the first possibility; then

$$
\left(\frac{l-1}{M}, \frac{l}{M}\right)=\left(\frac{d(l-1)}{N}, \frac{d l}{N}\right)
$$

contains an $N$-end.

Indeed there is, in the first place, an integer $r, d(l-1)<r<d l$, such that $r / N \in E$. For if

$$
x \in\left(\frac{d(l-1)}{N}, \frac{d l}{N}\right)
$$

belongs to $E$, we can certainly find such an $r$ with $\rho(x, r / N)<1 / N$. Then $r / N \in E$ since $N \in \mathscr{N}$ is always assumed. Now let $k$ be the least integer greater than or equal to $r$ such that $(k+1) / N \notin E$. Evidently $k \leqq d l-1$ since $l / M=d l / N \notin E$, and $k / N$ is an $N$-end.

Hence there is a $j \in S_{N}$ such that $k / N=j / N(\bmod 1)$. Since $d \mid N$ and $d \mid j$, it follows that $d \mid k$. But $d(l-1)<k<d l$, hence

$$
l-1<\frac{k}{d}<l,
$$

a contradiction.

The argument for the case when $((l / M),(l+1) / M)$ contains a point of $E$, is practically identical to this.

The next result implies our main theorem, and is useful in determining if a given set fails $(H)$. We shall need the following assumptions and notation:

Assume that $\sim E$ has infinitely many components, all with rational end points.

Let $l_{1}, l_{2}, \cdots$ be an enumeration of their lengths, with $l_{i}>l_{i+1}>0$ for all $i$. Evidently $\sum_{i=1}^{\infty} l_{i} \leqq 1$, so $l_{i} \rightarrow 0$ as $i \rightarrow \infty$.

Let $U_{i}$ be the union of all the components of $\sim E$ of lengths greater than or equal to $l_{i}, K_{i}$ the set of end points of these components, and $q_{i}$ the least common multiple of the denominators of the members of $K_{i}$, expressed in the lowest form.

THEOREM 4. If $E$ satisfies (H), then for infinitely many integers $i$, the following three conditions must hold simultaneously:

(a) $l_{i+1} \leqq \frac{1}{q_{i}}$.

(b) $2 l_{i+1}<l_{i}$.

(c) For each integer $j$ with $0 \leqq j<q_{i}$, if $j / q_{i} \notin E$, then $j / q_{i} \in U_{i}$. 
REMARK. If $E$ is a set for which condition (c) holds for infinitely many $i$, then $E$ satisfies $(H)$. Indeed, the boundary points of $U_{i}$ are all of the form $j / q_{i}$; thus if $i$ satisfies (c), $N=q_{i}$ satisfies (*). Moreover, $\left\{q_{i}: i\right.$ satisfies $\left.(c)\right\}$ will then be an infinite set. Indeed, $\left(1 / q_{i}\right) \leqq l_{i}$ for all $i$. Thus fixing $i$, if we choose $k>i$ such that $l_{k}<\left(1 / q_{i}\right)$, we have that $\left(1 / q_{k}\right)<\left(1 / q_{i}\right)$, so there are at most finitely many $j$ 's such that $q_{j}=q_{i}$.

Proof of Theorem 4. Assume that $E$ satisfies $(H)$, and fix $N \in \mathscr{N}$ with $N>1 / l_{1}$.

Then there is a unique $i$ such that $l_{i+1} \leqq(1 / N)<l_{i}$. By Lemma 1 , each member of $K_{i}$ is an $N$-end. Letting $E_{N}$ be as defined before the proof of Lemma 2, we thus have $U_{i} \subset \sim E_{N}$. Moreover, every component of $\sim E_{N}$ is a component of $\sim E$, of length greater than or equal to $2 / N$, by the definition of $E_{N}$. Thus, every component of $\sim E_{N}$ is of length greater than $l_{i+1}$, whence $\sim E_{N} \subset U_{i}$, and every $N$ end is a member of $K_{i}$, since it is an end point of a component of $\sim E$ of length greater than or equal to $l_{i}$. Thus $E_{N}=\sim U_{i}$ and the set of $N$-ends equals $K_{i}$. So every element in $K_{i}$ is of the form $j / N$, whence $q_{i} \mid N$, so $q_{i} \leqq N$, and thus (a) follows. Since $2 / N$ is less than or equal to the lengths of all the components of $\sim E_{N}=U_{i}$, it follows that $2 / N \leqq l_{i}$, whence (b) holds. Finally, it follows from the definition of $q_{i}$, that if $d$ is the greatest common divisor of $S_{N} \cup\{N\}$, then $q_{i}=N / d$ (where $S_{N}$ is defined in Lemma 3). Thus by Lemma 3, $q_{i} \in \mathscr{N}$, whence since $q_{i} \leqq N, E_{q_{i}} \supset E_{N}$ by Lemma 2 . So suppose that $j / q_{i} \in E$. Then

$$
\frac{j}{q_{i}} \notin E_{q_{i}}
$$

by the latter's definition, so $j / q_{i} \notin E_{N}$, whence $j / q_{i} \in U_{i}$, so (c) holds.

Finally since $\mathscr{N}$ is infinite, there must be infinitely many $i$ 's for which there exists an $N \in \mathscr{N}$ with $l_{i+1} \leqq 1 / N<l_{i}$, and consequently for which (a), (b), and (c) all hold.

Proof of the main theorem. Let $E$ satisfy $(H)$, and assume first that $\sim E$ has infinitely many components. Then by Lemma 1 , the end points of these components are all rational numbers, so Theorem 4 is applicable; thus condition (c) of that result holds for infinitely many integers $i$. Now fixing $i$ for which (c) holds, if $N>q_{i}$, then $q_{i} \mid N$; indeed, since $q_{i} \geqq 1 / l_{i}$, we obtain by Lemma 1 that every element of $K_{i}$ is an $N$-end, and thus expressable in the form $j / N$. Moreover, since the boundary points of $U_{i}$ are all of the form $j / q_{i}$, we obtain that $q_{i} \in \mathscr{N}$.

Thus simply let $j_{1}, j_{2}, \cdots$ be an enumeration of a subset of the 
$i$ 's satisfying (c), such that $q_{i_{r}}<q_{j_{r^{\prime}}}$ for all $r<r^{\prime}$. Then if we put $F_{i}=\sim U_{j_{i}}$ and $N_{i}=q_{j_{i}}$ for all $i, E=\bigcap_{i=1}^{\infty} F_{i}$ and (1)-(3) are satisfied for all $i$. We have also established that when $E$ satisfies $(H)$ and its complement, has infinitely many components then there exist $N_{1}<N_{2}<\cdots$ such that for all $i$ and $N$, if $N \geqq N_{i}$ then $N_{i} \mid N$.

Now if $E$ satisfies $(H)$ and $\sim E$ has only finitely many components, then by Lemma 1 , the boundary points of $E$ are all rational numbers. Let $M$ be the least common multiple of the denominators of these numbers expressed in the lowest form; then setting $N_{i}=2^{i-1} M$ and $F_{i}=E$ for all $i$, it is easily verified that (1)-(3) hold. We remark finally that if $\sim E$ has finitely many components with rational boundary points, then $E$ satisfies $(H)$, and in fact letting $M$ be as above, then for all $L \geqq M, L \in \mathscr{N}$ if and only if $M \mid L$. (Thus the statement ending the preceeding paragraph fails for $E$ 's such that $\sim E$ has finitely many components.)

We wish finitely to give some examples of sets which fail to satisfy $(H)$. If $\xi$ is a real number with $0<\xi<1 / 2, S_{\xi}$, the symmetric set of ratio $\xi$, consists of all numbers $x$ in $T$ such that

$$
x=(1-\xi) \sum_{j=0}^{\infty} \varepsilon_{i} \xi^{j}
$$

where $\varepsilon_{j}=0$ or 1 , all $j$. (See pages 13-15 of [2].)

Now $\xi$ is an end point of a component of $\sim S_{\xi}$, namely $(\xi, 1-\xi)$.

Hence if $\xi$ is irrational, then $S_{\xi}$ fails $(H)$ by Lemma 1 . If $\xi=1 / L$ for some integer $\mathrm{L}$, then it is well known that $S_{\xi}$ satisfies $(H)$. We shall show that if $\xi=p / q$, where $p$ and $q$ are relatively prime integers with $p>1$, then $S_{\xi}$ fails $(H)$.

Defining $l_{i}$ and $q_{i}$ for $E=S_{\xi}$, we have that $l_{i}=(1-2 \xi) \xi^{i-1}$ and $q_{i}=q^{i}$ for $i=1,2, \cdots$. (It follows from page 14 of [2] that all the end points of components of $U_{i}$ are of the form $l / q^{i}$ for some integer $l$; but $p^{i} / q^{i}$ is such an end point, and $p^{i}$ and $q^{i}$ are relatively prime.) Now if $l_{i+1} \leqq 1 / q_{i}$, then $(1-2(p / q))(p / q)^{i} \leqq 1 / q_{i}$, or $p^{i} \leqq q /(q-2 p)$; thus condition (a) of Theorem 4 will be violated for all $i$ sufficiently large.

\section{REFERENCES}

1. C.S. Herz, Spectral synthesis for the Cantor set, Proc. Nat. Acad. Sci. U.S.A. 42 (1956), 42-43.

2. J.P. Kahane, and R. Salem, Ensembles parfaits et series trigonometriques, Hermann, Paris, 1963.

Received January, 8, 1968. This research was supported by NSF-GP-5585. 


\section{PACIFIC JOURNAL OF MATHEMATICS}

\section{EDITORS}

\author{
H. ROYDEN \\ Stanford University \\ Stanford, California \\ R. R Phelps \\ University of Washington \\ Seattle, Washington 98105
}

\author{
J. Dugundui \\ Department of Mathematics \\ University of Southern California \\ Los Angeles, California 90007
}

RICHARD ARENS

University of California

Los Angeles, California 90024

\section{ASSOCIATE EDITORS}
E. F. BeCKENBACH
B. H. NeUmanN
F. WolF
K. YosidA

\section{SUPPORTING INSTITUTIONS}

\author{
UNIVERSITY OF BRITISH COLUMBIA \\ CALIFORNIA INSTITUTE OF TECHNOLOGY \\ UNIVERSITY OF CALIFORNIA \\ MONTANA STATE UNIVERSITY \\ UNIVERSITY OF NEVADA \\ NEW MEXICO STATE UNIVERSITY \\ OREGON STATE UNIVERSITY \\ UNIVERSITY OF OREGON \\ OSAKA UNIVERSITY \\ UNIVERSITY OF SOUTHERN CALIFORNIA
}

\author{
STANFORD UNIVERSITY \\ UNIVERSITY OF TOKYO \\ UNIVERSITY OF UTAH \\ WASHINGTON STATE UNIVERSITY \\ UNIVERSITY OF WASHINGTON

$* * * * *$
AMERICAN MATHEMATICAL SOCIETY
CHEVRON RESEARCH CORPORATION
TRW SYSTEMS
NAVAL WEAPONS CENTER

The Supporting Institutions listed above contribute to the cost of publication of this Journal, but they are not owners or publishers and have no responsibility for its content or policies.

Mathematical papers intended for publication in the Pacific Journal of Mathematics should be in typed form or offset-reproduced, double spaced with large margins. Underline Greek letters in red, German in green, and script in blue. The first paragraph or two must be capable of being used separately as a synopsis of the entire paper. It should not contain references to the bibliography. Manuscripts, in duplicate if possible, may be sent to any one of the four editors. Please classify according to the scheme of Math. Rev. 36, 1539-1546. All other communications to the editors should be addressed to the managing editor, Richard Arens, University of California, Los Angeles, California, 90024.

50 reprints are provided free for each article; additional copies may be obtained at cost in multiples of 50 .

The Pacific Journal of Mathematics is published monthly. Effective with Volume 16 the price per volume (3 numbers) is $\$ 8.00$; single issues, $\$ 3.00$. Special price for current issues to individual faculty members of supporting institutions and to individual members of the American Mathematical Society: $\$ 4.00$ per volume; single issues $\$ 1.50$. Back numbers are available.

Subscriptions, orders for back numbers, and changes of address should be sent to Pacific Journal of Mathematics, 103 Highland Boulevard, Berkeley, California, 94708.

PUBLISHED BY PACIFIC JOURNAL OF MATHEMATICS, A NON-PROFIT CORPORATION

Printed at Kokusai Bunken Insatsusha (International Academic Printing Co., Ltd.), 7-17. Fujimi 2-chome, Chiyoda-ku, Tokyo, Japan. 


\section{Pacific Journal of Mathematics}

Vol. 28, No. 3

May, 1969

Jon F. Carlson, Automorphisms of groups of similitudes over $F_{3} \ldots \ldots \ldots$

W. Wistar (William) Comfort, Neil Hindman and Stelios A. Negrepontis,

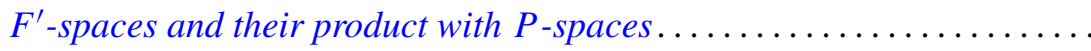

Archie Gail Gibson, Triples of operator-valued functions related to the unit

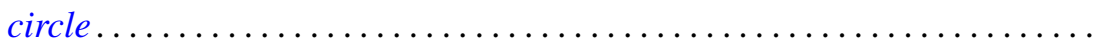

David Saul Gillman, Free curves in $E^{3}$

E. A. Heard and James Howard Wells, An interpolation problem for

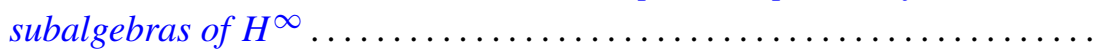

Albert Emerson Hurd, A uniqueness theorem for weak solutions of symmetric

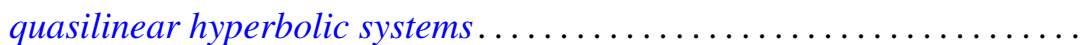

E. W. Johnson and J. P. Lediaev, Representable distributive Noether

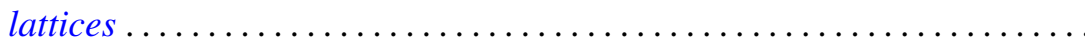

David G. Kendall, Incidence matrices, interval graphs and seriation in

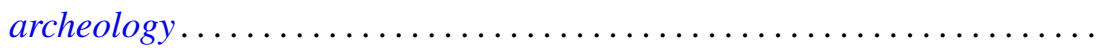
565

Robert Leroy Kruse, On the join of subnormal elements in a lattice ....... 571

D. B. Lahiri, Some restricted partition functions; Congruences modulo 3 .... 575

Norman D. Lane and Kamla Devi Singh, Strong cyclic, parabolic and conical differentiability........................................

William Franklin Lucas, Games with unique solutions that are

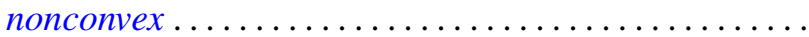

Eugene A. Maier, Representation of real numbers by generalized geometric series.

Daniel Paul Maki, A note on recursively defined orthogonal polynomials ...

Mark Mandelker, $F^{\prime}$-spaces and z-embedded subspaces ...

James R. McLaughlin and Justin Jesse Price, Comparison of Haar series with gaps with trigonometric series

Ernest A. Michael and A. H. Stone, Quotients of the space of irrationals ....

William H. Mills and Neal Zierler, On a conjecture of Golomb ...

J. N. Pandey, An extension of Haimo's form of Hankel convolutions ...

Terence John Reed, On the boundary correspondence of quasiconformal mappings of domains bounded by quasicircles...

Haskell Paul Rosenthal, A characterization of the linear sets satisfying Herz's criterion.

George Thomas Sallee, The maximal set of constant width in a lattice...

I. H. Sheth, On normaloid operators

James D. Stasheff, Torsion in BBSO ...

Billy Joe Thorne, A - P congruences on Baer semigroups.

Robert Breckenridge Warfield, Jr., Purity and algebraic compactness for

modules... 